\title{
Evolution of the $M_{\mathrm{BH}^{-}} \sigma$ and $M_{\mathrm{BH}^{-}}-L_{\text {bulge }}$ Relations
}

\author{
J.-H. Woo ${ }^{1}$, N. V. Bennert ${ }^{2}$, T. Treu ${ }^{2}$, M. Malkan ${ }^{3}$ and R. Blandford ${ }^{4}$ \\ ${ }^{1}$ Seoul National University, Republic of Korea \\ Email: woo@astro.snu.ac.kr \\ ${ }^{2}$ Dept. of Physics and Astronomy, University of California Santa Barbara, USA \\ Email: bennert, tt@physics.ucsb.edu \\ ${ }^{3}$ Dept. of Physics and Astronomy, University of California Los Angeles, USA \\ Email: malkan@astro.ucla.edu \\ ${ }^{4}$ Kavli Institute for Particle Astrophysics and Cosmology, Stanford University, USA \\ Email: rdb@slac.stanford.edu
}

\begin{abstract}
To constrain the origin of scaling relations between black hole mass and galaxy properties, i.e., stellar velocity dispersion and bulge luminosity, we investigate the evolution of scaling relations in the past 6 Gyrs. Over the last three years, we have obtained high signalto-noise ratio Keck spectra of $\sim 50$ intermediate luminosity broad-line AGNs at $z \sim 0.4$ and $z \sim 0.6$, to measure stellar velocity dispersion, and HST (ACS and NICMOS) images of the same objects ( $\sim 40$ so far), to measure bulge luminosity from the two-dimensional AGN-galaxy decomposition analysis. In this paper, we will summarize the main results on the $M_{\mathrm{BH}}-\sigma$ and $M_{\mathrm{BH}}$-bulge luminosity relations and their evolution to the present-day universe. The measured scaling relations show that the relations have evolved significantly in the past 6 billion years, and that black hole growth predates the final galaxy assembly.
\end{abstract}

Keywords. black hole physics, galaxies: evolution, galaxies: active

\section{Introduction}

In the local universe, supermassive black holes (BHs) seem to be ubiquitous at the centers of bulge-dominated galaxies, and the mass of the black holes $\left(M_{\mathrm{BH}}\right)$ correlates with global properties of their host galaxies, such as the stellar velocity dispersion $(\sigma)$ and luminosity of the spheroidal component $\left(L_{\text {bulge }}\right)$ (e.g., Kormendy \& Richstone 1995; Ferrarese \& Ford 2005). These relatively tight correlations indicate a close link between black hole growth and galaxy evolution (Ferrarese \& Merritt 2000; Gebhardt et al. 2000a). Understanding the origin of these scaling relations will provide a key to solving several astrophysical problems, such as the role of AGN feedback in suppressing star formation in massive galaxies (e.g., Di Matteo et al. 2005; Croton et al. 2006; Ciotti \& Ostiker 2007).

Various unified formation scenarios have been suggested in the literature to explain the origin of the scaling relations (e.g. Kauffmann \& Haehnelt 2000; Granato et al. 2004; Croton 2006; Robertson et al. 2006; Hopkins et al. 2007). In the standard cosmological model, spheroids grow by mergers of smaller galaxies while black holes grow by accreting surrounding matter. Depending on the relative timing and the efficiency of these two processes, the scaling relations between $M_{\mathrm{BH}}$ and velocity dispersion or spheroid luminosity could also evolve with cosmic time (e.g. Kauffmann \& Haenhelt 2000; Volonteri et al. 2003; Croton 2006; Hopkins et al. 2009).

One of the most powerful observational tests of the proposed scenarios is to directly measure the evolution of the scaling relations with redshift. We have investigated cosmic 
evolution of the $M_{\mathrm{BH}}-\sigma$ and $M_{\mathrm{BH}}-L_{\text {bulge }}$ relations in the last 6 Gyrs (see Treu et al. 2004; Woo et al. 2006; Treu et al. 2007; Woo et al. 2008). The limitation of our previous studies was that the range of black hole masses in our previous sample was limited to an order of magnitude, making it difficult to investigate the mass-dependency of the evolution. To overcome this limitation and to investigate various systematic effects, we chose a sample of lower black hole mass objects at the same redshift, for which we obtained high signal-to-noise Keck spectra and HST NICMOS images, enlarging the sample size and the dynamical range by almost a factor of two. The $M_{\mathrm{BH}}-\sigma$ relation results with a detailed analysis will be presented by Woo et al. (in preparation), while HST analysis on the $M_{\mathrm{BH}}-L_{\text {bulge }}$ has been recently presented by Bennert et al. (2009). Here, we briefly summarize the main results.

\section{The Present-Day $M_{\mathrm{BH}}-\sigma$ Relation for Active Galaxies}

To investigate cosmic evolution of scaling relations, first of all it is necessary to define the local $M_{\mathrm{BH}}-\sigma$ relation of active galaxies. Although active galaxies with measured reverberation black hole masses seem to follow the same $M_{\mathrm{BH}}-\sigma$ relation as non-AGN galaxies (Gebhardt et al. 2000b; Ferrarese et al. 2001), it has not been clearly shown whether the $M_{\mathrm{BH}}-\sigma$ relation of active galaxies has the same slope and scatter, due to the limited sample size and the black hole mass range of the local AGNs with reliable reverberation mass and stellar velocity dispersion measurements. Recently, Bentz et al. (2009b) reported new reverberation measurements for a sample of eight local Seyfert galaxies, and stellar velocity dispersions of these galaxies were measured by Woo et al. (2009).

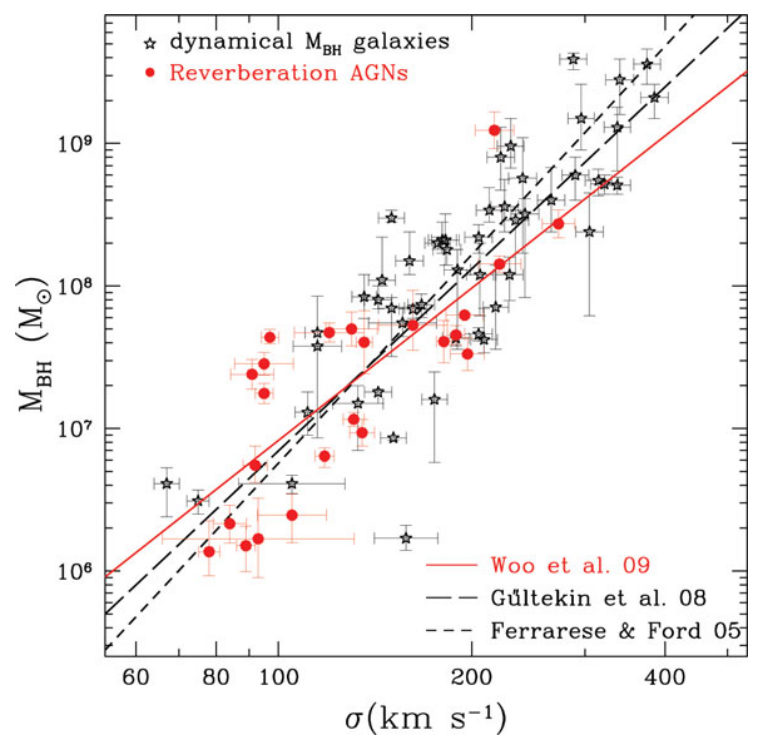

Figure 1. The $M_{\mathrm{BH}}-\sigma$ relation of active galaxies in the present-day universe from Woo et al. (2009). Active galaxies with reverberation black hole masses (circles) are compared with non-active galaxies with dynamical black hole masses from Gültekin et al. (2009; open stars). The solid line is the best-fit slope of the active galaxies (Woo et al. 2009) while the dashed and long-dashed lines are the best fits to the non-active galaxy samples from Ferrarese \& Ford (2005) and Gültekin et al. (2009), respectively. Active and non-AGN galaxies follow a consistent $M_{\mathrm{BH}}-\sigma$ relation with a smilar slope and scatter. 
In Figure 1, we show the $M_{\mathrm{BH}}-\sigma$ relation of all available reverberation-mapped AGNs with measured stellar velocity dispersion (filled circles), with reverberation $M_{\mathrm{BH}}$ ranging from $10^{6}$ to $10^{9} M_{\odot}$. The solid line is the best fit slope of $3.55 \pm 0.60$ for the reverberation sample. Active galaxies in the present-day universe follow a $M_{\mathrm{BH}}-\sigma$ relation that is consistent with that of non-AGN galaxies. Also, the intrinsic scatter of the relation for the active galaxies is $\sim 0.43 \mathrm{dex}$, which is close to that of non-AGN galaxy $M_{\mathrm{BH}}-\sigma$ relation $\left(0.44 \pm 0.06\right.$; Gültekin et al. 2009). These results indicate that the same $M_{\mathrm{BH}}-\sigma$ relation holds in the present-day universe, regardless of AGN activity.

\section{The Distant Active Galaxy Sample}

\subsection{Sample Selection}

To study the scaling relations at higher redshifts, we selected a sample of $\sim 50$ intermediate-luminosity broad-line AGNs, where the fraction of stellar light is large enough to measure stellar velocity dispersion with high $S / N$ spectra. At the same time, virial $M_{\mathrm{BH}}$ can be obtained from the integrated properties of the broad-line region (BLR) using the same spectrum. To minimize the systematic uncertainties related to sky subtraction and atmospheric absorption corrections, we chose specific redshift windows where the relevant emission and absorption lines $(\mathrm{H} \beta, \mathrm{Mg} \mathrm{Ib}$, and $\mathrm{Fe} \mathrm{I})$ fall in clean regions of the atmosphere. We chose relatively low redshift windows at $z=0.36 \pm 0.01$ and $z=0.57 \pm 0.01$ in order to securely measure the stellar velocity dispersion of AGN host galaxies. However the corresponding look-back time is $\sim 4$ Gyrs and $\sim 6$ Gyrs, respectively, which is a significant fraction of the age of the universe.

\subsection{Measurements}

Stellar velocity dispersion: By comparing the observed host galaxy spectra with stellar templates broadened with a Gaussian velocity in spectral regions around $\mathrm{Mg} \operatorname{Ib} \lambda 5175$ and Fe I $\lambda 5270$, we measured the stellar velocity dispersion of 13 new objects, using high $S / N$ Keck spectra. Combined with our previous sample (see Woo et al. 2006, 2008 for our previous results), the enlarged sample consists of 32 broad-line AGNs and the dynamical range is increased over two orders of magnitude $\left(10^{7}<M_{\mathrm{BH}} / M_{\odot}<10^{9}\right)$.

Bulge luminosity: To measure bulge luminosity, two-dimensional surface photometry was performed with the HST NICMOS data using the GALFIT software (Peng et al. 2002). We used point source, bulge, and disk components for fitting, and if needed, an additional component with a Sersic profile with $n=0.5$ was added to model a bar. We used the same method for measuring bulge luminosity as we have used for our ACS data analysis (see Treu et al. 2007). Combining our new $L_{\text {bulge }}$ measurement for 20 objects, we assembled a total sample of 40 broad-line AGNs to study the $M_{\mathrm{BH}}-L_{\text {bulge }}$ relation.

At the same time, we reanalyzed the archival HST ACS data for the local reverberation sample of 19 objects to match the procedure adopted for higher- $z$ sample. Thus, we can study the evolution with consistently measured bulge luminosity.

Black hole mass: $M_{\mathrm{BH}}$ was estimated using the "virial" method, assuming that the kinematics of the BLR trace the gravitational field of the central black hole (Wandel et al. 1999; Kaspi et al. 2005; Bentz et al. 2006; McGill et al. 2008; Bentz et al. 2009a). The virial coefficient is obtained by requiring that local AGN hosts and quiescent galaxies obey the same $M_{\mathrm{BH}}-\sigma$ relation (Woo et al. 2009). In practice, $M_{\mathrm{BH}}$ was estimated using the equation

$$
\log M_{\mathrm{BH}}=8.58+2 \log \left(\frac{\sigma_{\mathrm{H} \beta}}{3000 \mathrm{~km} \mathrm{~s}^{-1}}\right)+0.518 \log \left(\frac{L_{5100}}{10^{44} \mathrm{erg} \mathrm{s}^{-1}}\right),
$$


where $\sigma_{\mathrm{H} \beta}$ is the second moment of the $\mathrm{H} \beta$ line profile and $L_{5100}$ is the nuclear luminosity at $5100 \AA$ measured from our HST analysis.

\section{Cosmic Evolution of Scaling Relations}

In this section, we compare our new sample of distant galaxies and the local reverber-

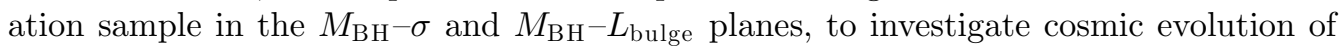
scaling relations.

\subsection{The Evolution of the $M_{\mathrm{BH}}-\sigma$ Relation}

Combining our previous sample of active galaxies presented by Woo et al. $(2006 ; 2008)$ and additional AGNs with newly measured stellar velocity dispersions and single-epoch black hole mass estimates, we have an enlarged sample of 32 broad-line AGNs with black hole mass range of $10^{7}-10^{9} M_{\odot}$. In Figure 2, we compare the $M_{\mathrm{BH}}-\sigma$ relation of this distant galaxy sample with that of the local non-AGN galaxies.

Confirming our previous result, the $M_{\mathrm{BH}}-\sigma$ relation of the enlarged sample, particularly with lower $M_{\mathrm{BH}}$ objects $\left(M_{\mathrm{BH}}<10^{8} M_{\odot}\right)$, shows a systematic offset from the local relationship, indicating cosmic evolution of the relation. At fixed black hole mass, distant active galaxies appear to have smaller bulge stellar velocity dispersions, implying that these bulges have to significantly grow in the past $4-6$ billion years if they have to be on the local relationship by $z \sim 0$.

To quantify the evolution, we calculate the offset from the local relationship in black hole mass units, and present them as a function of redshift in Figure 2b. On average, the offset at $z \sim 0.4$ and $z \sim 0.6$ is approximately 0.5 dex, implying recent evolution of bulges at these mass scales. While individual galaxies show large scatter, the average offset implies that black hole growth predates the final assembly of spheroids.
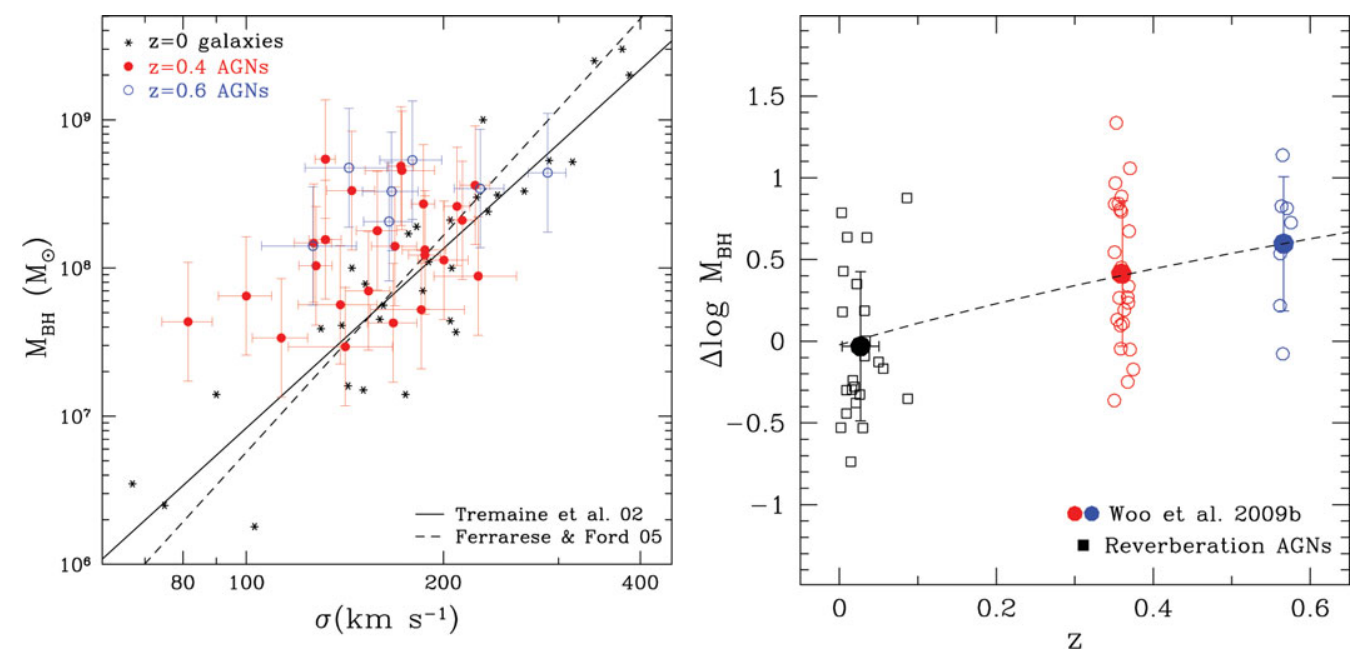

Figure 2. Left: The $M_{\mathrm{BH}}-\sigma$ relation for distant Seyferts (circles) and local quiescent galaxies (black dots), compared with the best fit relations from Tremaine et al. (2002) (solid line) and Ferrarese \& Ford (2005) (dashed line). Right: Offset from the local $M_{\mathrm{BH}}-\sigma$ relation. Large solid points with error bars represent the average and rms scatter for the three samples. The best linear fit to the data is shown as a solid line. The average offset of the $z \sim 0.4$ and the $z \sim 0.6$ points is $\sim 0.5$ dex in $M_{\mathrm{BH}}$. Note that $M_{\mathrm{BH}}$ is estimated consistently with the same virial coefficient and therefore the relative position of the three samples along the $y$-axis is independent of the virial coefficient. 


\subsection{The Evolution of the $M_{\mathrm{BH}}-L_{\text {bulge Relation }}$}

The $M_{\mathrm{BH}}-L_{\text {bulge }}$ relation shows a similar trend in cosmic evolution. In Figure 3 , we compare distant active galaxies with the local reverberation AGNs. The distant active galaxies and the local AGNs cover approximately the same range in spheroid luminosity, but the average $M_{\mathrm{BH}}$ is higher for the distant Seyferts. The average offset with respect to the local reverberation sample corresponds to $\Delta \log M_{\mathrm{BH}}=0.14 \pm 0.07 \pm 0.20$, when we include an intrinsic error as a free parameter and marginalize over it.

\section{Discussion and Conclusions}

The two scaling relations show a significant offset from the local relationship, indicating cosmic evolution of scaling relations. If the local $M_{\mathrm{BH}}-\sigma$ and $M_{\mathrm{BH}}-L_{\text {bulge }}$ relations are the end point of black hole-galaxy coevolution, then our distant galaxies have to significantly grow their bulges in the next 4-6 billion years. The exact amount of bulge-mass growth depends on the size evolution and stellar mass-to-light ratios. Nevertheless, our results indicate a general trend that black hole growth predates the final assembly of spheroids, as other studies on cosmic evolution at high redshift consistently indicate (Peng et al. 2002; Jahnke et al. 2009; Merloni et al. 2009).

However, our results do not imply that all galaxies at $z \sim 0.4-0.6$ have to grow by large amounts since there is mass-dependency on the offset. For example, when we include a quasar sample selected from a similar redshift range $(z \sim 0.3-0.4)$ with measured host galaxy luminosity using $H S T$ archival data, these more massive black holes and their host galaxies do not show a clear offset from the local $M_{\mathrm{BH}}-L_{\text {bulge }}$ relation. This may be a sign of downsizing of cosmic evolution. More massive galaxies with early-type morphologies are already on the relationship, while less massive and later-type galaxies

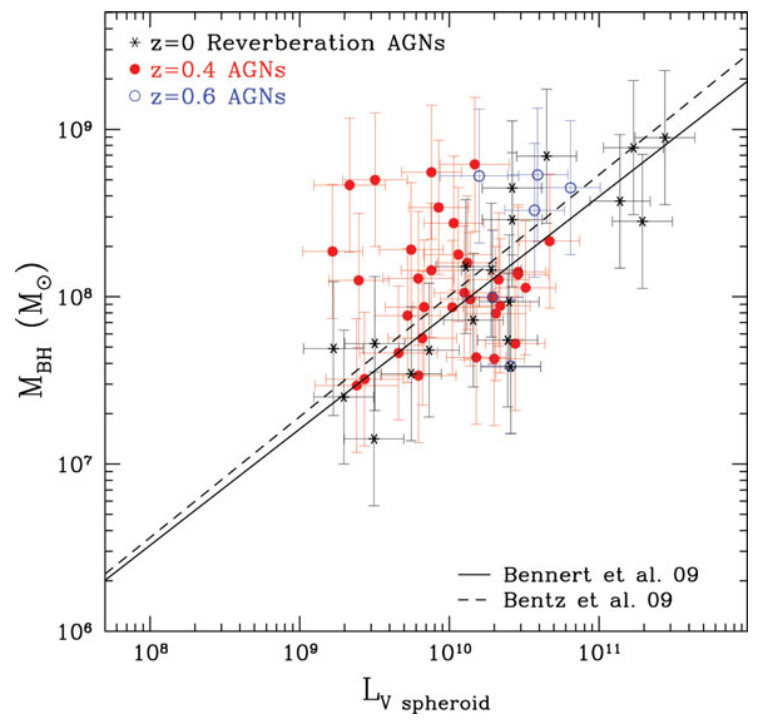

Figure 3. The $M_{\mathrm{BH}}-L_{\mathrm{bulge}, V}$ relation of active galaxies. Distant active galaxies (filled circle: $z \sim 0.4$, open circle: $z \sim 0.6$ ) are compared with the local reverberation AGNs studied by Bentz et al. (2009a), but reanalyzed by Bennert et al. (2009) (black stars). Solid and dashed lines are the best fit to the local reverberation sample from Bennert et al. (2009) and Bentz et al. (2009a), respectively. At fixed black hole mass, the distant active galaxy bulges have lower luminosity than the local active galaxies. For direct comparison, $L_{\mathrm{bulge}}$ of the distant Seyferts has been passively evolved to $z=0$. 
are still evolving to the local relationship, and the critical mass scale, under which the relation shows systematic offset from the local relationship, decreases with time.

Selection bias may be present in our sample, possibly resulting in the observed evolution of the scaling relations. However, our Monte Carlo simulation study indicates that only 0.1 dex offset could be atributted to selection effects (Treu et al. 2007; see also Lauer et al. 2007 for similar results), suggesting that selection bias cannot entirely account for the offset. Larger samples of AGNs with measured $M_{\mathrm{BH}}, \sigma$, and host-galaxy properties are needed both locally and at high redshift to improve the understanding of selection effects.

The observed evolution can be qualitatively explained if our Seyferts undergo a single collisional merger with a disk-dominated system between $z \sim 0.4-0.6$ and today. A single merger could increase the spheroid mass by transporting stellar mass from the progenitors' disks. At the same time, this process would add younger stars to the spheroid (either from the merging disks or from newly formed stars), thus increasing stellar velocity dispersion and bulge luminosity more efficiently than the growth of black holes. Numerical simulations including realistic prescriptions for star formation, AGN activity, and mass loss will be needed to see if these mergers preserve $R_{\mathrm{e}}$ and $M_{\mathrm{sph}} / L_{B}$, which will confirm that black holes completed their growth before their host galaxies at these mass scales.

\section{References}

Bennert, V. N., et al. 2009, ApJ, in press

Bentz, M. C., et al. 2006, ApJ, 644, 133

Bentz, M. C., et al. 2009a, ApJ, 697, 160

Bentz, M. C., et al. 2009b, ApJ, 705, 199

Ciotti, L., \& Ostriker, J. P. 2007, ApJ, 665, 1038

Croton, D. J. 2006, MNRAS, 369, 1808

Di Matteo, T., et al. 2005, Nature, 433, 604

Ferrarese, L., \& Merritt, D. 2000, ApJ, 539, L9

Ferrarese, L., et al. 2001, ApJ, 555, L79

Gebhardt, K., et al. 2000a, ApJ, 539, L13

Gebhardt, K., et al. 2000b, ApJ, 543, L5

Hopkins, P. F., et al. 2007, ApJ, 669, 45

Hopkins, P. F., et al. 2009, ApJ, 669, 45

Jahnke, K., et al. 2009, ApJ, 706, 215

Kaspi, S., et al. 2005, ApJ, 629, 61

Kauffmann, G. \& Haehnelt, M. 2000, MNRAS, 311, 576

Merloni, A., et al. 2009, ApJ, in press

McGill, K., Woo, J.-H., Treu, T., \& Malkan, M. A. 2008, ApJ, 673, 703

Peng, C., et al. 2002, AJ, 124, 266

Tremaine, S., et al. 2002, ApJ, 574, 740

Treu, T., Woo, J.-H., Malkan, M. A., \& Blandford, R. D. 2007, ApJ, 667, 117

Volonteri, M., Haardt, F., \& Madau, P. 2003, ApJ, 582, 559

Woo, J.-H., Treu, T, Malkan, M. A., \& Blandford, R. D. 2006, ApJ, 645, 900

Woo, J.-H., Treu, T, Malkan, M. A., \& Blandford, R. D. 2008, ApJ, 681, 295

Woo, J.-H., et al. 2009, ApJ, submitted 